\title{
BMJ Open Sexual minority status and suicidal behaviour among Chinese adolescents: a nationally representative cross-sectional study
}

\author{
Yeen Huang, ${ }^{1}$ Pengsheng Li, ${ }_{1}^{1}$ Lan Guo, ${ }^{1}$ Xue Gao, ${ }^{2}$ Yan $X u,{ }^{2}$ Guoliang Huang, ${ }^{2}$ \\ Xueqing Deng, ${ }^{1,3}$ Ciyong $\mathrm{Lu}^{1,3}$
}

To cite: Huang Y, Li P, Guo L, et al. Sexual minority status and suicidal behaviour among Chinese adolescents: a nationally representative crosssectional study. BMJ Open 2018;8:e020969. doi:10.1136/ bmjopen-2017-020969

- Prepublication history for this paper is available online. To view these files, please visit the journal online (http://dx.doi org/10.1136/bmjopen-2017020969).

$\mathrm{YH}$ and PL contributed equally.

Received 5 December 2017

Revised 22 June 2018

Accepted 29 June 2018

Check for updates

(c) Author(s) (or their employer(s)) 2018. Re-use permitted under CC BY-NC. No commercial re-use. See rights and permissions. Published by BMJ.

${ }^{1}$ Department of Medical Statistics and Epidemiology, School of Public Health, Sun Yat-sen University, Guangzhou, China

${ }^{2}$ Center for Adverse Drug Reactions Monitoring of Guangdong, Guangzhou, China ${ }^{3}$ Guangdong Provincial Key Laboratory of Food, Nutrition and Health, Guangzhou, China

Correspondence to

Dr Ciyong Lu;

luciyong@mail.sysu.edu.cn

\section{ABSTRACT}

Objectives Suicidality among sexual minority adolescents has generated worldwide concern in recent decades, and previous Western studies have demonstrated that sexual minority status is associated with adolescent suicidality. However, whether this association exists in Chinese adolescents remains largely unknown. This study aimed to estimate the associations between sexual minority status and suicidal behaviour among Chinese adolescents.

Design Cross-sectional survey.

Setting A total of 506 high schools in 7 provinces of China.

Participants A total of 150822 students in grades 7-12 who completed the questionnaires (response rate of $95.9 \%$ ) were included.

Main outcome measures Suicidal ideation and suicide attempts were used to measure suicidal behaviour, and sexual attraction (opposite sex, same sex or both sex) was used as a measure for sexual minority status.

Results of the 150822 adolescents analysed, $4.1 \%$ selfreported as sexual minorities and $17.3 \%$ were unsure. Compared with heterosexual and unsure adolescents, same-sex romantic attraction (SSA) and both-sex romantic attraction (BSA) adolescents reported a higher prevalence of past-year suicidal ideation (SSA: $21.6 \%$ for males and $30.4 \%$ for females; BSA: $34.7 \%$ for males and $42.3 \%$ for females) and suicide attempts (SSA: $6.9 \%$ for males and $8.9 \%$ for females; BSA: $12.2 \%$ for males and $10.9 \%$ for females). After adjustment for covariates, SSA and BSA adolescents were more likely to have past-year suicidal ideation and suicide attempts than their heterosexual and unsure peers. BSA adolescents reported the highest risk of suicidal ideation (males: adjusted OR (AOR) $2.42,95 \% \mathrm{Cl}$ 2.03 to 2.88; females: AOR 2.61, 95\% $\mathrm{Cl} 2.41$ to 2.82) and suicide attempts (males: AOR $3.83,95 \% \mathrm{Cl} 2.85$ to 5.14 ; females: AOR 2.59, $95 \% \mathrm{Cl} 2.19$ to 3.06 ).

Conclusions Our study suggested that Chinese sexual minority adolescents were at increased risk of suicidality, and those with BSA had an especially high risk in this population. These findings emphasised the urgent need to develop targeted interventions to effectively address suicide-related problems among Chinese sexual minority adolescents.
Strengths and limitations of this study

- Our study estimated the prevalence of suicidality and examined the association between sexual minority status and suicidal behaviour among Chinese adolescents.

- A large-scale, nationally representative sample provided sufficient statistical power, and between-groups analyses were conducted.

- Due to the nature of the cross-sectional data, interpretation of the direction of the observed associations is limited.

- Our study sample included only students attending school and did not include adolescents who dropped out of school or were absent from school on the day the survey was administered.

\section{INTRODUCTION}

Suicidal behaviour, which includes suicidal ideation, suicide attempts and completed suicide, has raised concerns about the health of sexual minority adolescents (ie, adolescents who experience same-sex attraction, engage in same-sex sexual behaviour or self-identify as gay, lesbian, bisexual) in recent decades. Minority stress theory ${ }^{1}$ suggests that difficulties in dealing with minority stressors (prejudice, discrimination and stigma) associated with same-sex orientation may lead to substance abuse, ${ }^{2}$ depression ${ }^{3}$ and even suicide $^{4}$ among sexual minorities. Compared with their heterosexual peers, sexual minority adolescents have been identified in numerous studies as a high-risk group for suicidal behaviour. ${ }^{5}$ Regarding the associations of sexual minority status with suicidal behaviour, most related studies have been conducted in Western or developed countries. Previous findings from the Youth Risk Behavior Survey in the USA showed that approximately $42.8 \%$ and $29.4 \%$ of sexual minority adolescents reported having past-year suicidal ideation 
and suicide attempts, respectively. ${ }^{6}$ Two longitudinal studies from the USA and Norway showed that sexual minority adolescents were two and four times more likely, respectively, to have attempted suicide in the past-year than their heterosexual peers. ${ }^{5} 7$ Moreover, a previous systematic review indicated that bisexual individuals from developed countries have the highest levels of suicide risk among sexual minorities. ${ }^{8}$ Those findings thus identified sexual minority status as a risk factor for adolescent suicidality in Western or developed countries.

As the largest low/middle-income country with a high suicide rate, China accounts for $21 \%$ of the world's population and $30 \%-40 \%$ of the world's suicides. ${ }^{9}$ Although numerous studies have focused on suicide risk in Chinese adolescents, ${ }^{10}{ }^{11}$ little attention has been devoted to sexual minorities. Previous studies in China have shown that $4.6 \%-12.6 \%$ of gay adults have reported lifetime suicide attempts, ${ }^{12} 13$ and sexual minority youth from Taipei were almost two times as likely to have past-year suicidal ideation as their heterosexual peers. ${ }^{14}$ It is well known that the cultural background of Western countries is different from that of Asian countries, especially China, where Confucianism has been an influence for thousands of years and families and social climates exert intense pressure on individuals to marry and have children to maintain their family lineages. ${ }^{15}$ Thus, sexual minorities are recognised as a major impediment to continuing the family line and maintaining a family's reputation, ${ }^{16}$ and sexual minorities in China may experience more minority stressors and higher levels of mental and behavioural problems. ${ }^{17} 18$ However, there is a paucity of research exploring the associations between sexual minority status and suicidal behaviour among mainland Chinese adolescents; whether this well-known increased suicide risk for sexual minorities can also be found in Chinese adolescents remains largely unknown.

Therefore, we conducted this nationally representative large-scale study to estimate the prevalence of suicidality among Chinese sexual minority adolescents, to evaluate the associations between sexual minority status and suicidal ideation and attempts, to investigate whether these associations vary in different sexual minority statuses, and to provide suggestions for effective policy-making and developing intervention strategies for governmental public health organisations.

\section{METHODS}

\section{Study design and participants}

We used data from the 2015 School-Based Chinese Adolescents Health Survey (SCAHS) ${ }^{19}{ }^{20}$ an ongoing, large-scale health-related behaviour survey among Chinese adolescents (grades 7-12). The SCAHS has been conducted every 2 years since 2007, and the 2015 survey was the most recent version conducted in seven Chinese provinces. ${ }^{21}$

In the 2015 SCAHS, students were selected via a fourstage, stratified-cluster, random-sampling method. In stage 1, all 34 province-level regions in China were divided into four regional strata (East China, West China, South China and North China), and then two representative provinces from each regional stratum were selected by simple randomisation (only one province from East China). In stage 2, cities in each representative province were divided into three economic strata (high level, middle level and low level) by per capita GDP. Based on the proportions of these three types of cities, six cities were randomly selected from each representative province. In stage 3 , schools were divided into three categories: junior high schools (ie, grades 7-9), senior high schools (ie, grades 10-12) and vocational high schools (ie, grades 7-12). Based on the proportions of these three types of schools, four junior high schools, four senior high schools and four vocational high schools were randomly selected from each representative city (506 schools agreed to participate in this study). In stage 4 , two classes were randomly selected from each grade within the selected schools, and all available students in the selected classes were invited to participate in this study voluntarily. In total, 150822 students completed the questionnaires (response rate of 95.9\%).

\section{Participants involvement and data collection}

All students from the chosen classes were given a standardised self-administered questionnaire which was developed by research team on the day of the survey, to be completed in the classroom during a normal class period (40 or $45 \mathrm{~min}$ ). To protect student privacy, the questionnaire was completed by each student participant anonymously without the presence of teachers or other school personnel (to avoid any potential information bias). After collecting the questionnaires from students, investigators would check the questionnaires carefully and contact with the student timely when they found important missing data. The investigators were all trained and quality control was carried out during data collection. All data were collected from November 2014 to January 2015.

\section{Measures \\ Suicidal ideation and suicide attempts}

Suicidal ideation was defined as responding 'one or more times' to the following question: 'During the past 12 months, how many times did you seriously consider attempting suicide?' Suicide attempts were assessed by asking students to respond to the following question with zero, once or more: 'During the past 12 months, how many times did you actually attempt suicide? 2223

\section{Sexual minority status}

The number of transsexuals among sexual minority adolescents is still very $\operatorname{low}^{24}$ and was difficult to investigate in our school-based survey, and this minority group was therefore not included in this study. Sexual minority status was measured by asking students the following question regarding sexual attraction: 'In a romantic relationship, what kind of person are you attracted to?' Response 
options included the following: (1) opposite sex, (2) same sex, (3) equally opposite sex and same sex and (4) unsure. ${ }^{25}{ }^{26}$ Respondents were classified as the following categories: (1) heterosexual, (2) same-sex romantic attraction (SSA), (3) both-sex romantic attraction (BSA) and (4) unsure. Students who belonged to categories (2) and (3) were classified as sexual minorities.

\section{Demographic variables}

Factors previously reported to be associated with suicidal behaviour in sexual minority adolescents were taken into consideration. ${ }^{4527}$ Demographic variables included sex, age, academic pressure, household socioeconomic status (HSS), current smoking, current drinking and bullying experience.

After reading a brief definition of bullying from the Olweus Bully/Victim Questionnaire, adolescents were asked the following question: 'How often have you been bullied (kicked, intentionally excluded from participating, made fun of with sexual jokes, etc) at school in the past 30 days? ${ }^{28}$ Answers were given on a three-point scale as follows: (1) never, (2) sometimes or rarely (one or two times) or (3) often (more than three times). Students reporting a frequency of 'often' in the past 30 days were classified as being bullied. ${ }^{29}$ Academic pressure was assessed based on students' self-rating about their school work; responses were coded as follows: (1) none, (2) less or (3) medium or great. HSS was measured by asking about the student's perception of his or her household's current socioeconomic status; responses were coded as follows: (1) very good, (2) good and (3) fair or poor. Current smoking was measured by asking the following question: 'During the past 30 days, on how many days did you smoke cigarettes?' Students who selected answers indicating 1 or more days were classified as current smokers. ${ }^{30}{ }^{31}$ Current drinking was assessed with the following question: 'During the past 30 days, on how many days did you drink alcohol?' Students who selected answers indicating 1 or more days were classified as current drinkers. ${ }^{32}$

\section{Statistical analysis}

Prevalence estimates and logistic regression analyses used appropriate sampling weights (adjusting for students' grade, sex and school location) and estimation procedures that accounted for the complex sampling design. Taylor series estimation methods were used to obtain proper SE estimates. First, descriptive analyses were conducted to describe the demographic characteristics and prevalence of suicidality. Second, Rao-Scott $\mathrm{X}^{2}$ tests and one-way analysis of variance were used to compare the differences in demographic characteristics and suicide rates between groups. Third, univariate logistic regression models were performed to explore the associations between sexual minority status and suicidal ideation and suicide attempts without the confounding effects of sex. Additional multivariate models were sequentially adjusted for age, academic pressure, HSS, current smoking, current drinking and bullying experience. Based on previously reported studies, ${ }^{33} 34$ age, sociofamily environment (eg, academic pressure and HSS), unhealthy behaviours (eg, smoking and drinking) and school environment (eg, bullying experience) were associated with suicidal behaviours in China, and all were added as covariates to determine the independent associations between sexual minority status and suicidality. Missing data accounted for less than $3.1 \%$ for all relevant variables and were eliminated from the statistical analysis. ORs and 95\% CIs were obtained from logistic regression models. $\mathrm{P}$ values less than 0.05 were considered statistically significant (tested two sided) for regression analysis. The alpha level for paired comparison was set by Bonferroni correction. All statistical analyses were conducted using SAS V.9.4 (SAS Institute).

\section{RESULTS}

\section{Demographic characteristics}

The characteristics of the students are shown in table 1 . Of the total sample, the mean (SE) age of the adolescents was $15.1(0.4)$ years old; $51.8 \%$ were males and $48.2 \%$ were females. Among these adolescents, $4.1 \%$ self-reported as sexual minorities, $17.3 \%$ as unsure and $78.6 \%$ as heterosexual. Compared with their unsure and heterosexual peers, sexual minority adolescents were more likely to be females $(\mathrm{p}<0.001)$, to come from a family with poor socioeconomic status $(p<0.001)$ and to report medium or great academic pressure $(\mathrm{p}<0.001)$. Additionally, sexual minority adolescents were also more likely to report smoking, alcohol use and school bullying experiences during the past 30 days than their unsure and heterosexual peers.

\section{Prevalence of suicidality by sexual minority status}

As shown in table 2, for male adolescents, the weighted prevalence of past-year suicidal ideation was more frequently reported in sexual minority (SSA: $21.6 \%$; BSA: $34.7 \%)$ adolescents than in their heterosexual $(14.50 \%)$ and unsure $(11.7 \%)$ peers, and BSA students reported the highest rate of past-year suicidal ideation. The weighted prevalence of past-year suicide attempts was higher in sexual minority (SSA: $6.9 \%$; BSA: $12.2 \%$ ) and unsure $(3.1 \%)$ adolescents than in their heterosexual peers $(2.2 \%)$, and BSA students reported the highest rate of past-year suicide attempts.

As for female adolescents, the weighted prevalence of past-year suicidal ideation was higher in sexual minority (SSA: $30.4 \%$; BSA: $42.3 \%$ ) adolescents than in their heterosexual $(18.8 \%)$ and unsure $(14.9 \%)$ peers, with BSA students having the highest prevalence. The weighted prevalence of past-year suicide attempts was higher in sexual minority adolescents (SSA: $8.9 \%$; BSA: $10.9 \%)$ than in their heterosexual $(3.1 \%)$ and unsure $(3.2 \%)$ peers, and BSA adolescents reported the highest rate of past-year suicide attempts. 
Table 1 Demographic characteristics of participants by sexual minority status among Chinese adolescents ( $\mathrm{n}=150822)$

\begin{tabular}{|c|c|c|c|c|c|c|}
\hline & Total & Heterosexual & Sexual minorities* & Unsure & & \\
\hline Variable & No $(\%)$ & No (\%) & No (\%) & No (\%) & $\chi^{2} / \mathbf{F}$ & $P$ values \\
\hline Total & $150822(100.00)$ & 116774 (78.6) & $6685(4.1)$ & $27363(17.3)$ & & \\
\hline Sex & & & & & 182.9 & $<0.001$ \\
\hline Male & $72409(51.8)$ & $57343(52.8)$ & $2483(41.4)$ & $12583(49.7)$ & & \\
\hline Female & $78413(48.2)$ & $59431(47.2)$ & $4202(58.6)$ & $14780(50.3)$ & & \\
\hline Age (year) $\dagger$ & $15.1(0.4)$ & $15.3(0.5)$ & $15.1(0.4)$ & $14.1(0.3)$ & 754.33 & $<0.001$ \\
\hline Academic pressure & & & & & 567.69 & $<0.001$ \\
\hline None & $23387(15.8)$ & 17051 (14.9) & $1060(16.1)$ & $5276(19.8)$ & & \\
\hline Less & $69359(46.5)$ & $53061(46.1)$ & 2648 (39.9) & $13650(49.8)$ & & \\
\hline Medium or great & $58076(37.7)$ & $46662(39.0)$ & $2977(44.0)$ & $8437(30.4)$ & & \\
\hline HSS & & & & & 204.56 & $<0.001$ \\
\hline Very good & $30766(22.7)$ & $22812(21.8)$ & $1456(24.2)$ & 6498 (26.6) & & \\
\hline Good & $90894(60.3)$ & $71306(61.1)$ & $3748(55.4)$ & $15840(57.4)$ & & \\
\hline Fair or poor & $29162(17.0)$ & $22656(17.1)$ & $1481(20.4)$ & 5025 (16.0) & & \\
\hline Current smoking & & & & & 288.12 & $<0.001$ \\
\hline No & $143032(94.6)$ & $110194(94.1)$ & $6212(93.0)$ & 26626 (97.3) & & \\
\hline Yes & $7790(5.4)$ & $6580(5.9)$ & $473(7.0)$ & $737(2.7)$ & & \\
\hline Current drinking & & & & & 818.05 & $<0.001$ \\
\hline No & $126765(84.0)$ & 96909 (82.9) & $5072(76.6)$ & $24784(90.5)$ & & \\
\hline Yes & $24057(16.0)$ & 19865 (17.1) & $1613(23.4)$ & $2579(9.5)$ & & \\
\hline Bullying experience & & & & & 57.58 & $<0.001$ \\
\hline No & $138523(91.6)$ & 107767 (92.0) & 5668 (83.9) & $25088(91.3)$ & & \\
\hline Yes & $12299(8.4)$ & 9007 (8.0) & $1017(16.1)$ & $2275(8.7)$ & & \\
\hline
\end{tabular}

All numbers were unweighted, whereas all percentages were adjusted for sampling weights.

*Sexual minorities included adolescents who reported same-sex or both-sex romantic attraction.

†Age data are presented as the means (SE).

HSS, household socioeconomic status.

\section{Associations between sexual minority status and suicidal ideation and suicide attempts}

As shown in table 3, for male adolescents, unadjusted analyses (model 1) showed that SSA and BSA adolescents had a higher risk of suicidal ideation and suicide attempts than their heterosexual peers. After adjustment for academic pressure, HSS, current smoking, current drinking and bullying experience (model 2), SSA (adjusted OR (AOR) 1.56, 95\% CI 1.26 to 1.94 ) and BSA (AOR 2.42, 95\% CI 2.03 to 2.88) adolescents were more likely to report suicidal ideation than their heterosexual and unsure peers. Compared with heterosexual peers, SSA (AOR 3.13, 95\% CI 2.28 to 4.28), BSA (AOR 3.83, $95 \%$ CI 2.85 to 5.14 ) and unsure (AOR $1.55,95 \%$ CI 1.24 to 1.94) male adolescents were more likely to have suicide attempts.

As for female adolescents, unadjusted analyses (model 1) showed that SSA and BSA adolescents had a higher risk of suicidal ideation and suicide attempts than their heterosexual and unsure peers. After adjustment for academic pressure, HSS, current smoking, current drinking and bullying experience (model 2). SSA (AOR 1.42, 95\% CI
1.30 to 1.56 ) and BSA (AOR 2.61, 95\% CI 2.41 to 2.82) adolescents were more likely to report suicidal ideation than their heterosexual and unsure peers. Compared with heterosexual and unsure peers, SSA (AOR 1.97, 95\% CI 1.43 to 2.70) and BSA (AOR 2.59, 95\% CI 2.19 to 3.06) female students were more likely to have suicide attempts.

Moreover, in both male and female adolescent sexual minorities, differences in the risk of suicide attempts were more pronounced than differences in the risk of suicidal ideation compared with those in heterosexual and unsure peers, and BSA adolescents reported the highest risk of suicide attempts.

\section{DISCUSSION}

As in many studies in Western or developed countries, we determined that Chinese sexual minority adolescents had a higher risk of suicidal ideation and suicide attempts than their heterosexual and unsure peers; being a BSA male or female was associated with an increased risk of suicidality. To our knowledge, this study is the first to use 
Table 2 Prevalence of past-year suicidal ideation and suicide attempts by sexual minority status among Chinese adolescents $(n=150822)$

\begin{tabular}{|c|c|c|c|c|c|c|c|c|}
\hline & \multicolumn{4}{|l|}{ Males ( $\mathrm{N}=72$ 409) } & \multicolumn{4}{|c|}{ Females ( $\mathrm{N}=78$ 413) } \\
\hline & Heterosexual=1 & SSA $=2$ & $B S A=3$ & Unsure $=4$ & Heterosexual=5 & SSA $=6$ & $B S A=7$ & Unsure $=8$ \\
\hline & No (\%) & No (\%) & No (\%) & No (\%) & No (\%) & No (\%) & No (\%) & No (\%) \\
\hline No & $48572(85.5)$ & $795(78.4)$ & $919(65.3)$ & 11067 (88.3) & 47941 (81.2) & 706 (69.6) & $1839(57.7)$ & $12527(85.1)$ \\
\hline Yes & $8771(14.5)$ & $243(21.6)$ & $526(34.7)$ & $1516(11.7)$ & $11490(18.8)$ & $309(30.4)$ & $1348(42.3)$ & $2253(14.9)$ \\
\hline No & 55991 (97.8) & $962(93.1)$ & $1267(87.8)$ & $12184(96.9)$ & 57552 (96.9) & $923(91.1)$ & $2820(89.1)$ & $14318(96.8)$ \\
\hline Yes & $1352(2.2)$ & $76(6.9)$ & $178(12.2)$ & 399 (3.1) & $1879(3.1)$ & $92(8.9)$ & $367(10.9)$ & 462 (3.2) \\
\hline
\end{tabular}

All numbers were unweighted, whereas all percentages were adjusted for sampling weights.

The alpha level for paired comparison was set at $p=0.0083$ after Bonferroni correction.

Paired comparison for suicidal ideation: Paired comparison for suicide attempts:

$2>1, \chi^{2}=51.42, \mathrm{P}<0.001 ; 6>5, \chi^{2}=78.41, \mathrm{P}<0.001 ; 2>1, \chi^{2}=105.29, \mathrm{P}<0.001 ; 6>5, \chi^{2}=110.22, \mathrm{P}<0.001$.

$3>1, \chi^{2}=471.59, \mathrm{P}<0.001 ; 7>5, \chi^{2}=978.63, \mathrm{P}<0.001 ; 3>1, \chi^{2}=551.67, \mathrm{P}<0.001 ; 7>5, \chi^{2}=610.42, \mathrm{P}<0.001$.

$3>2, \chi^{2}=47.69, \mathrm{P}<0.001 ; 7>6, \chi^{2}=45.29, \mathrm{P}<0.001 ; 3>2, \chi^{2}=16.42, \mathrm{P}<0.001 ; 7>6, \chi^{2}=4.76, \mathrm{P}=0.029$.

$4<1, \chi^{2}=86.74, \mathrm{P}<0.001 ; 8<5, \chi^{2}=131.20, \mathrm{P}<0.001 ; 4>1, \chi^{2}=27.95, \mathrm{P}<0.001 ; 8>5, \chi^{2}=0.05, \mathrm{P}=0.824$.

$4<2, \chi^{2}=110.08, \mathrm{P}<0.001 ; 8<6, \chi^{2}=161.47, \mathrm{P}<0.001 ; 4<2, \chi^{2}=49.09, \mathrm{P}<0.001 ; 8<6, \chi^{2}=98.96, \mathrm{P}<0.001$;.

$4<3, \chi^{2}=618.07, \mathrm{P}<0.001 ; 8<7, \chi^{2}=1197.33, \mathrm{P}<0.001 ; 4<3, \chi^{2}=274.99, \mathrm{P}<0.001 ; 8<7, \chi^{2}=419.29, \mathrm{P}<0.001$.

$\mathrm{BSA}$, both-sex romantic attraction; SSA, same-sex romantic attraction.

nationally representative data to explore the associations between sexual minority status and suicidal behaviour among Chinese adolescents.

Consistent with previous studies, ${ }^{4-6}$ our results additionally revealed that sexual minority adolescents had a higher prevalence of both suicidal ideation and suicide attempts than their heterosexual peers. Compared with a cross-sectional survey conducted with sexual minority youths from three Asian cities (Hanoi, Shanghai and Taipei), ${ }^{14}$ the prevalence of past-year suicidal ideation and suicide attempts in our sexual minority samples was similar to that in Taipei samples but higher than that in Shanghai and Hanoi samples. This variation in results may derive from the different sample sources and age structures. Our findings provide population-based evidence of the prevalence of suicidal behaviour among Chinese sexual minorities, which is useful for identifying adolescents who may be at high risk of suicide.

Furthermore, we found that, compared with their heterosexual peers, Chinese sexual minority adolescents had increased risk of suicidality after stratification by sex. To our knowledge, because of their insufficient sample sizes, most previous studies combined individuals with different sexual minority statuses into one category without considering sex stratification, which may obscure the estimates of suicide risk among high-risk adolescents. ${ }^{35} 36$ This study is the first to use a nationally representative and largescale sample to explore the associations between sexual minority status and suicidal behaviour among Chinese adolescents grouped according to sexual minority status and stratified by sex. Our results are consistent with a previous systematic review that reported elevated risks of past-year suicide attempts in homosexual and bisexual adolescents, especially males. ${ }^{37}$ One possible mechanism to explain these associations is the experience of minority stress. ${ }^{1}$ According to the minority stress model, sexual minority individuals may experience minority stressors (eg, prejudice events, internalised homophobia), which are related to lower well-being and higher levels of suicidal ideation. ${ }^{38}$ Previous results from a 2011 National School Climate Survey indicated that more than $60 \%-80 \%$ of sexual minority students reported being verbally harassed and that $40 \%$ of students experienced physical violence at school during the past-year. ${ }^{39}$ Experiences such as being threatened or injured are directly related to suicidality among sexual minority adolescents. ${ }^{40}$ In current Chinese society, stigma against non-heterosexual individuals persists and a large portion of the general population shows intolerant attitudes towards sexual minorities. ${ }^{41} \mathrm{In}$ our study, we also found that Chinese sexual minorities have a higher prevalence of bullying experiences than their heterosexual peers. Therefore, a negative school environment may play a potential role in the associations between sexual minority status and suicidal behaviour among Chinese adolescents.

In line with previous studies, ${ }^{37} 42$ our study found that sexual orientation-associated differences were more pronounced for suicide attempts than for suicidal ideation, and BSA adolescents showed a higher risk of suicide attempts than their SSA, unsure and heterosexual peers. Several reasons may explain the more severe forms of suicidality among the bisexual group. First, bisexual individuals may experience additional forms of minority stress from both gays/lesbians and heterosexuals, and the various forms of biphobia and monosexism can create emotional and cognitive dysfunction that may lead to depression, anxiety or even suicide attempts. ${ }^{143}$ Second, bisexual individuals are pervasively invisible in society. 
Table 3 Associations of sexual minority status with past-year suicidal ideation and suicide attempts among Chinese adolescents $(n=150822)$

\begin{tabular}{|c|c|c|c|c|c|c|}
\hline & \multicolumn{3}{|c|}{ Model 1* } & \multicolumn{3}{|c|}{ Model 2† } \\
\hline & OR & $95 \% \mathrm{Cl}$ & $P$ values & AOR & $95 \% \mathrm{Cl}$ & $P$ values \\
\hline \multicolumn{7}{|l|}{ Suicidal ideation } \\
\hline \multicolumn{7}{|l|}{ Males } \\
\hline Heterosexual & 1.0 & & & 1.0 & & \\
\hline SSA & 1.62 & 1.30 to 2.03 & $<0.001$ & 1.56 & 1.26 to 1.94 & $<0.001$ \\
\hline BSA & 3.13 & 2.59 to 3.79 & $<0.001$ & 2.42 & 2.03 to 2.88 & $<0.001$ \\
\hline Unsure & 0.78 & 0.67 to 0.91 & $<0.001$ & 0.78 & 0.69 to 0.87 & $<0.001$ \\
\hline \multicolumn{7}{|l|}{ Females } \\
\hline Heterosexual & 1.0 & & & 1.0 & & \\
\hline SSA & 1.88 & 1.67 to 2.11 & $<0.001$ & 1.42 & 1.30 to 1.56 & $<0.001$ \\
\hline BSA & 3.15 & 2.93 to 3.39 & $<0.001$ & 2.61 & 2.41 to 2.82 & $<0.001$ \\
\hline Unsure & 0.75 & 0.60 to 0.94 & $<0.001$ & 0.71 & 0.61 to 0.83 & $<0.001$ \\
\hline \multicolumn{7}{|l|}{ Suicide attempts } \\
\hline \multicolumn{7}{|l|}{ Males } \\
\hline Heterosexual & 1.0 & & & 1.0 & & \\
\hline SSA & 3.29 & 2.43 to 4.47 & $<0.001$ & 3.13 & 2.28 to 4.28 & $<0.001$ \\
\hline BSA & 6.25 & 4.46 to 8.76 & $<0.001$ & 3.83 & 2.85 to 5.14 & $<0.001$ \\
\hline Unsure & 1.42 & 1.07 to 1.90 & $<0.001$ & 1.55 & 1.24 to 1.94 & $<0.001$ \\
\hline \multicolumn{7}{|l|}{ Females } \\
\hline Heterosexual & 1.0 & & & 1.0 & & \\
\hline SSA & 3.13 & 2.36 to 4.15 & $<0.001$ & 1.97 & 1.43 to 2.70 & $<0.001$ \\
\hline BSA & 3.89 & 3.13 to 4.83 & $<0.001$ & 2.59 & 2.19 to 3.06 & $<0.001$ \\
\hline Unsure & 1.04 & 0.75 to 1.44 & 0.824 & 1.03 & 0.80 to 1.34 & 0.531 \\
\hline
\end{tabular}

*Unadjusted.

†Adjusted for age, academic pressure, household socioeconomic status, current smoking, current drinking and bullying experience. AOR, adjusted OR; BSA, both-sex romantic attraction; SSA, same-sex romantic attraction.

Heterosexual and homosexual people have mutual interests in maintaining the primacy of monosexual assumptions and binary sexual orientation, which may contribute to an internalised sense of belief that bisexuals do not belong to any particular sexual minority group. The lack of a sense of belonging may be one of the factors contributing to suicide attempts among bisexual people. ${ }^{843}$ Third, lack of social and healthcare support was more commonly reported among bisexual individuals than among their homosexual and heterosexual peers, and this lack of support made bisexuals feel more socially isolated and vulnerable to chronic stress and led to an increased risk of suicide. ${ }^{44}$ Moreover, our results reported that $17.3 \%$ of adolescents reported being unsure about sexual romantic attraction, which is higher than that reported in previous Western research. ${ }^{45}$ One potential explanation is that the unsure category may include many adolescents who did not understand the question about sexual minority status or were unwilling to disclose their sexual orientation. In accordance with a previous systematic review, ${ }^{37}$ the risk of past-year suicide attempts was smaller for unsure adolescents than for sexual minorities in our study.
However, previous studies reported that unsure adolescents may show same-sex attraction or behaviors ${ }^{46}$ and thus may experience minority stress (eg, bullying victimisation), ${ }^{47}$ leading to health disparities such as depression, anxiety ${ }^{48}$ and suicidal ideation. ${ }^{49}$ In contrast to previous studies, our findings showed that unsure adolescents had no increased risk of suicidal ideation compared with heterosexual peers. One possible reason explaining the discrepant findings could be the much larger proportion of unsure adolescents in our Chinese sample. Therefore, further research to explore the prevalence and mechanisms of suicidality among unsure adolescents is needed.

Chinese sexual minorities suffer from minority stressors due to discrimination, homophobia and other conditions in the social environment impacted by traditional Chinese culture (which is rooted in Confucian philosophies). ${ }^{50}$ Confucianism emphasises the continuation of the family line and filial piety to protect the family's reputation and lineage (eg, prior to 2016, the One-Child Policy; from 2016 to the present, the Two-Child Policy). ${ }^{16}$ Although attitudes towards Chinese sexual minorities have become more positive in particular populations (eg, 
younger or highly educated people),${ }^{51}$ a large proportion of the Chinese population still holds negative attitudes towards sexual minorities. Same-sex orientation is still considered to conflict with traditional values and associated with prejudice and stigma in the current Chinese social context. ${ }^{52}$ These negative attitudes towards sexual minorities and minority stressors that they experience have been linked to high levels of mental and behavioural problems, such as depression and suicide attempts. ${ }^{17}{ }^{18} \mathrm{In}$ this study, our findings suggested that sexual minority status was associated with suicidal behaviour among Chinese adolescents and that BSA individuals were the highest risk group in this population. Therefore, the following appropriate interventions for suicidality among Chinese sexual minority adolescents are recommended. First, government and policy-makers should establish a set of nationwide policies and programmes to provide a significant source of support for sexual minorities and to reduce the homophobia arising from societal/structural homophobia and rigid gender roles. Second, schools and related public health organisations should formulate policies to prevent students from experiencing minority stressors (eg, being bullied at school) to reduce discrimination and create a generally positive school climate. Third, online resources (eg, online sex education websites) should be integrated to provide more relevant information and education, which may help to foster a more tolerant and open atmosphere towards sexual minorities. Fourth, families and communities should provide social support (eg, Gay-Straight Alliance groups, and Parents and Friends of Lesbians and Gays) to promote acceptance of sexual minority orientation ${ }^{53}$ and reduce pressure from traditional values and norms embedded in Confucianism (eg, filial piety and family responsibilities).$^{51}$ Fifth, practitioners (eg, psychologists, psychiatrists, counsellors and social workers) should specifically focus on the group that is at particularly high risk of suicidality (ie, BSA adolescents) with a weaker collective identity. Developing early and effective suicide-related preventive interventions (eg, treatment of depression, anxiety and their comorbidities) ${ }^{13}$ can help improve mental well-being in high-risk sexual minority adolescents.

Some noteworthy limitations should be considered when interpreting the results of this study. First, due to the cross-sectional design, it is difficult to make causal inferences. Second, our study used a structured selfrating questionnaire to collect data. Although self-reporting is a common and accepted method in sexuality research on adolescents, we could not completely rule out the possibility of recall bias and misclassification bias. Third, our study sample included only students attending school and did not include adolescents who dropped out of school or were absent from school on the day the survey was administered; suicidality may be more common among sexual minority students who were absent, possibly leading to underestimation of the sexual orientation disparities in our study. Fourth, we used the item on SSA to measure sexual minority status, and the results might not be comparable to those in other studies using sexual orientation as a measurement. However, our measure is particularly appropriate for surveying the health of sexual minority adolescents ${ }^{45}$ and is more likely to capture a broad range of adolescents who have 'come out' or acknowledged romantic attraction but may not have adopted a homosexual identity, ${ }^{5}$ which may help to identify that high-risk population. Fifth, gender dysphoria/transgender status was not measured in our study because sexual minority status was assumed using a binary definition of sex; although this way of definition is common in current scientific practice, we were unable to evaluate the experiences of suicidality in this minority group. Despite these limitations, the primary strengths of our study include its nationally representative and largescale sample of Chinese adolescents, providing sufficient statistical power and potentially avoiding oversampling of the sexual minority population. Furthermore, to the best of our knowledge, our study is the first study investigating the risk for suicidal behaviour among Chinese sexual minority adolescents in a representative sample.

\section{CONCLUSIONS}

Increased suicide risk among sexual minority adolescents has become a major global health concern. However, few related studies have been conducted among Chinese adolescents. The findings from our study suggested that Chinese sexual minority adolescents had a higher risk of suicidal behaviour and that BSA adolescents have the highest risk of suicidality regardless of sex. Based on our study results, conducting early detection and intervention programmes for Chinese sexual minorities (especially BSA individuals) is suggested to more effectively and appropriately prevent suicide-related problems. Future studies that focus on the risk factors, mechanisms and interventions of suicidal behaviour in Chinese sexual minority adolescents are warranted.

Acknowledgements The authors would like to thank local health professionals, the department of education and participating schools for their assistance and support. In addition, we express our gratitude to all the participants and investigators for assistance in data collection.

Contributors CL conceptualised and designed the study, reviewed and revised the manuscript and approved the final manuscript as submitted. YH and PL conceptualised and designed the study, coordinated and supervised the data collection, carried out the initial analyses, drafted the initial manuscript, and approved the final manuscript as submitted. They contributed equally to this study. LG carried out the analyses and interpreted the data, reviewed and revised the manuscript and approved the final manuscript as submitted. XG, YX, GH and XD designed the data collection instruments, coordinated and supervised the data collection, reviewed and revised the manuscript, and approved the final manuscript as submitted. All authors approved the final manuscript as submitted and agreed to be accountable for all aspects of the work.

Funding This work was supported by the National Natural Science Foundation of China (Grant number 81673252) and the Natural Science Foundation of Guangdong Province, China (Grant number 2014A030313174).

Competing interests None declared.

Patient consent Parental/guardian consent obtained.

Ethics approval This study was approved by the Sun Yat-Sen University School of Public Health Institutional Review Board. 
Provenance and peer review Not commissioned; externally peer reviewed.

Data sharing statement № additional data are available.

Open access This is an open access article distributed in accordance with the Creative Commons Attribution Non Commercial (CC BY-NC 4.0) license, which permits others to distribute, remix, adapt, build upon this work non-commercially, and license their derivative works on different terms, provided the original work is properly cited, appropriate credit is given, any changes made indicated, and the use is non-commercial. See: http://creativecommons.org/licenses/by-nc/4.0/.

\section{REFERENCES}

1. Meyer IH. Prejudice, social stress, and mental health in lesbian, gay, and bisexual populations: conceptual issues and research evidence. Psychol Bull 2003;129:674-97.

2. Marshal MP, Friedman MS, Stall R, et al. Sexual orientation and adolescent substance use: a meta-analysis and methodological review. Addiction 2008;103:546-56.

3. Marshal MP, Dietz LJ, Friedman MS, et al. Suicidality and depression disparities between sexual minority and heterosexual youth: a metaanalytic review. J Adolesc Health 2011;49:115-23.

4. Remafedi G, French S, Story M, et al. The relationship between suicide risk and sexual orientation: results of a population-based study. Am J Public Health 1998;88:57-60.

5. Russell ST, Joyner K. Adolescent sexual orientation and suicide risk: evidence from a national study. Am J Public Health 2001;91:1276-81.

6. Kann L, Olsen Emily O'Malley, McManus T, et al. Sexual Identity, Sex of Sexual Contacts, and Health-Related Behaviors Among Students in Grades 9-12 - United States and Selected Sites, 2015. MMWR. Surveillance Summaries 2016;65:1-202.

7. Wichstrøm L, Hegna K. Sexual orientation and suicide attempt: a longitudinal study of the general Norwegian adolescent population. $J$ Abnorm Psychol 2003;112:144-51.

8. Salway T, Ross LE, Fehr CP, et al. A Systematic Review and MetaAnalysis of Disparities in the Prevalence of Suicide Ideation and Attempt Among Bisexual Populations. Arch Sex Behav 2018:1-23.

9. Phillips MR, Li X, Zhang Y, et al. Suicide rates in China, 1995-99. The Lancet 2002;359:835-40.

10. Liu XC, Chen H, Liu ZZ, et al. Prevalence of suicidal behaviour and associated factors in a large sample of Chinese adolescents. Epidemiol Psychiatr Sci 2017:1-10.

11. Guo L, Xu Y, Deng J, et al. Association between sleep duration, suicidal ideation, and suicidal attempts among Chinese adolescents: The moderating role of depressive symptoms. J Affect Disord 2017;208:355-62.

12. Chen $\mathrm{H}$, Li Y, Wang L, et al. Causes of suicidal behaviors in men who have sex with men in China: a national questionnaire survey. BMC Public Health 2015;15:1-4.

13. Mu H, Li Y, Liu L, et al. Prevalence and risk factors for lifetime suicide ideation, plan and attempt in Chinese men who have sex with men. BMC Psychiatry 2016;16:1-10.

14. Lian Q, Zuo X, Lou C, et al. Sexual orientation and risk factors for suicidal ideation and suicide attempts: a multi-centre cross-sectional study in three Asian cities. J Epidemiol 2015;25:155-61.

15. Zhan HJ, Montgomery RJ V. Gender and elder care in China: The influence of filial piety and structural constraints. Gender \& Society 2003;17:209-29.

16. Hsu MH, Waters JA. Filial Piety and sexual prejudice in Chinese culture. Attitude Measures 2001:11.

17. Feng Y, Wu Z, Detels R. Evolution of men who have sex with men community and experienced stigma among men who have sex with men in Chengdu, China. J Acquir Immune Defic Syndr 2010;53:S98-S103.

18. Liu JX, Choi K. Experiences of social discrimination among men who have sex with men in Shanghai, China. AIDS Behav 2006;10:25-33.

19. Wang H, Deng J, Zhou X, et al. The nonmedical use of prescription medicines among high school students: a cross-sectional study in Southern China. Drug Alcohol Depend 2014;141:9-15.

20. Guo L, Xu Y, Deng J, et al. Non-medical use of prescription pain relievers among high school students in China: a multilevel analysis. BMJ Open 2015;5:e007569.

21. Guo L, Xu Y, Deng J, et al. Associations between childhood maltreatment and non-medical use of prescription drugs among Chinese adolescents. Addiction 2017;112:1600-9.

22. Guo L, Xu Y, Deng J, et al. Association between nonmedical use of prescription drugs and suicidal behavior among adolescents. JAMA Pediatr 2016;170:971-8.

23. Woods ER, Lin YG, Middleman A, et al. The associations of suicide attempts in adolescents. Pediatrics 1997;99:791-6.
24. Arcelus J, Bouman WP, Van Den Noortgate W, et al. Systematic review and meta-analysis of prevalence studies in transsexualism. Eur Psychiatry 2015;30:807-15.

25. Gay and Lesbian Medical Association. Bisexual (LGB) Youth Sexual Orientation Measurement Work Group. Measuring sexual orientation of young people in health research. San Francisco, CA: Gay and Lesbian Medical Association, 2003.

26. Li P, Huang Y, Guo L, et al. Sexual attraction and the nonmedical use of opioids and sedative drugs among Chinese adolescents. Drug Alcohol Depend 2018;183:169-75.

27. Shields JP, Whitaker K, Glassman J, et al. Impact of victimization on risk of suicide among lesbian, gay, and bisexual high school students in San Francisco. J Adolesc Health 2012;50:418-20.

28. Olweus D. The revised Olweus bully/victim questionnaire. Bergen, Norway: University of Bergen, 1996.

29. Wu J, He Y, Lu C, et al. Bullying behaviors among Chinese schoolaged youth: a prevalence and correlates study in Guangdong Province. Psychiatry Res 2015;225:716-22.

30. Acierno R, Kilpatrick DG, Resnick HS, et al. Assault, PTSD, family substance use, and depression as risk factors for cigarette use in youth: findings from the National Survey of Adolescents. J Trauma Stress 2000;13:381-96.

31. Kandra KL, McCullough A, Ranney L, et al. Support among middle school and high school students for smoke-free policies, North Carolina, 2009. Prev Chronic Dis 2013;10:675-81.

32. Huang R, Ho SY, Wang MP, et al. Sociodemographic risk factors of alcohol drinking in Hong Kong adolescents. J Epidemiol Community Health 2016;70:374-9.

33. Li Y, Li Y, Cao J, et al. Factors associated with suicidal behaviors in mainland China: a meta-analysis. BMC Public Health 2012;12:1-13.

34. Dai J, Chiu HF, Conner KR, et al. Suicidal ideation and attempts among rural Chinese aged 16-34 years--socio-demographic correlates in the context of a transforming China. J Affect Disord 2011;130:438-46.

35. Swannell S, Martin G, Page A. Suicidal ideation, suicide attempts and non-suicidal self-injury among lesbian, gay, bisexual and heterosexual adults: Findings from an Australian national study. Aust N Z J Psychiatry 2016;50:145-53.

36. de Graaf R, Sandfort TG, ten Have M. Suicidality and sexual orientation: differences between men and women in a general population-based sample from the Netherlands. Arch Sex Behav 2006;35:253-62

37. Plöderl M, Tremblay P. Mental health of sexual minorities. A systematic review. Int Rev Psychiatry 2015;27:367-85.

38. Baams L, Grossman AH, Russell ST, et al. Minority stress and mechanisms of risk for depression and suicidal ideation among lesbian, gay, and bisexual youth. Dev Psychol 2015;51:688-96.

39. Kosciw JG, Greytak EA, Bartkiewicz MJ, et al. The 2011 National School Climate Survey: The experiences of lesbian, gay, bisexual and transgender youth in our nation's schools. New York: GLSEN, 2012.

40. Bontempo DE, D'Augelli AR. Effects of at-school victimization and sexual orientation on lesbian, gay, or bisexual youths' health risk behavior. J Adolesc Health 2002;30:364-74.

41. Kwok DK, Wu J. Chinese attitudes towards sexual minorities in Hong Kong: Implications for mental health. Int Rev Psychiatry 2015;27:444-54.

42. Pompili M, Lester D, Forte $A$, et al. Bisexuality and suicide: a systematic review of the current literature. J Sex Med 2014;11:1903-13.

43. Hatzenbuehler ML. How does sexual minority stigma "get under the skin"? A psychological mediation framework. Psychol Bull 2009;135:707-30.

44. Koh AS, Ross LK. Mental health issues: a comparison of lesbian, bisexual and heterosexual women. J Homosex 2006;51:33-57.

45. Kann L, Olsen EO, McManus T, et al. Sexual identity, sex of sexual contacts, and health-risk behaviors among students in grades 9-12--youth risk behavior surveillance, selected sites, United States, 2001-2009. MMWR Surveill Summ 2011;60:1-133.

46. Igartua K, Thombs BD, Burgos G, et al. Concordance and discrepancy in sexual identity, attraction, and behavior among adolescents. J Adolesc Health 2009;45:602-8.

47. Birkett M, Russell ST, Corliss HL. Sexual-orientation disparities in school: the mediational role of indicators of victimization in achievement and truancy because of feeling unsafe. Am J Public Health 2014;104:1124-8.

48. Oswalt SB, Wyatt TJ. Sexual orientation and differences in mental health, stress, and academic performance in a national sample of U.S. college students. J Homosex 2011;58:1255-80.

49. Zhao $\mathrm{Y}$, Montoro R, Igartua K, et al. Suicidal ideation and attempt among adolescents reporting "unsure" sexual identity or heterosexual identity plus same-sex attraction or behavior: 
forgotten groups? J Am Acad Child Adolesc Psychiatry 2010;49:104-13.

50. Kwok DK. School experience of Chinese sexual minority students in Hong Kong. J LGBT Youth 2016:13:378-96.

51. Chi X, Hawk ST. Attitudes toward same-sex attraction and behavior among chinese university students: tendencies, correlates, and gender differences. Front Psychol 2016;7:1-9.
52. Lin K, Button DM, Su M, et al. Chinese college students' attitudes toward homosexuality: exploring the effects of traditional culture and modernizing factors. Sexuality Research and Social Policy 2016:13:158-72.

53. Whitaker K, Shapiro VB, Shields JP, et al. School-Based Protective Factors Related to Suicide for Lesbian, Gay, and Bisexual Adolescents. J Adolesc Health 2016;58:63-8. 